\title{
A Practical Approach for Geographic Prioritization and Targeting of Insecticide-Treated Net Distribution Campaigns During a Public Health Emergency
}

\author{
Alyssa Young ( $\nabla$ ayoung1@tulane.edu ) \\ 6929 \\ Will Eaton \\ Tulane University School of Public Health and Tropical Medicine \\ Matt Worges \\ Tulane University School of Public Health and Tropical Medicine \\ Honelgn Hiruy \\ Tulane University School of Public Health and Tropical Medicine \\ Kolawole Maxwell \\ Malaria Consortium
}

Tulane University School of Public Health and Tropical Medicine https://orcid.org/0000-0002-1703-

Bala Mohammed Audu

National Malaria Elimination Programme

Madeleine Marasciulo

Malaria Consortium

Charles Nelson

Malaria Consortium

James Tibenderana

Malaria Consortium

Tarekegn A. Abeku

Malaria Consortium

\section{Research}

Keywords: Plasmodium falciparum, malaria, vector control, prioritization scheme, intervention targeting, insecticide-treated nets, indoor residual spraying, COVID-19

Posted Date: May 11th, 2021

DOl: https://doi.org/10.21203/rs.3.rs-500247/v1 
License: (c) (i) This work is licensed under a Creative Commons Attribution 4.0 International License. Read Full License 


\section{Abstract}

\section{Background}

The use of data in targeting of malaria control efforts is becoming increasingly adopted by national malaria control programmes and is essential for optimal use of resources. This is especially important during public health emergencies such as the COVID-19 pandemic which resulted in reallocation of funding towards containment efforts. This work provides a practical mechanism for prioritizing geographical areas for insecticide-treated net (ITN) distribution during a public health emergency or situations of limited resources. We adopt a GIS-based weighted approach to categorize and rank administrative units based on data inputs that are widely available and can be applied in various country contexts where Plasmodium falciparum transmission is reported.

Methods

P. falciparum-specific intervention and prevailing risk factors were used to rank administrative units in Nigeria for prioritization during ITN distributions. Each factor was assigned a unique weight and was multiplied by a value, typically ranging from 1 to 4 , based on natural groupings inherent in the data, or the presence or absence of a given intervention. Risk scores for each factor were then summated to generate a composite unique risk score for each administrative unit. This risk score was then translated into a prioritization map which ranks each administrative unit from low to high priority in terms of ITN distribution targeting in situations of limited resources.

Results

A major component that influenced the prioritization scheme was previous ITN depth of coverage, as obtained and spatially interpolated from DHS survey data. The geospatial heterogeneity found among input risk factors suggests that a range of variables and covariates - outside of those that characterize malaria transmission exclusively - should be considered when using data to inform ITN distributions. The approach resulted in identification of regions that should be prioritized in upcoming ITN distributions.

\section{Conclusion}

The presented approach provides a tool for prioritizing regions for ITN distributions. It serves as a base upon which a wider range of vector control interventions could be targeted. Its value added can be found in its potential for application in multiple country contexts, expediated timeframe for producing outputs, and its use of systematically collected malaria indicators in informing prioritization.

\section{Background}

The use of data in informing malaria control efforts is increasingly being adopted by National Malaria Control Programmes (NMCPs). Data used to inform malaria interventions has expanded to encompass a variety of data points and covariates that are no longer limited to reported malaria case counts ${ }^{1-3}$. 
Improvements in the specificity and resolution in which interventions are targeted may not only increase the impact of a particular intervention but also facilitate optimal use of limited resources. The use of new types of insecticide-treated nets (ITNs) and chemicals for indoor residual spraying (IRS) due to insecticide resistance has resulted in increased vector control costs, which would make prioritization necessary. The success achieved in reducing malaria incidence in many endemic countries following the widescale implementation of effective interventions over the past two decades has produced less homogenous transmission levels compared to situations in the past. Different areas require different combinations of interventions to optimize the use of available resources and to address heterogeneity and increasing costs of universal coverage of vector control. Furthermore, prioritization is especially relevant during public health emergencies such as the current severe acute respiratory syndrome 2 (SARS-CoV-2, "COVID19 ") pandemic as many malaria control programmes have had to reallocate funding and resources towards COVID-19 containment efforts and safety measures ${ }^{4}$. The COVID-19 pandemic has not only affected countries in Sub-Saharan Africa directly by increasing strain on an already overburdened healthcare infrastructure, but also indirectly through cessation or delay in other disease control activities, including those essential in preventing and treating malaria cases ${ }^{4}$.

The objective of this work was to develop a practical, data-driven mechanism for prioritizing geographical areas for distribution of ITNs amidst public health emergency caused by the COVID-19 pandemic. Whereas the use of routine surveillance and malaria intervention coverage data is typically recommended when targeting malaria interventions, we aimed to create a simple approach that could be easily interpreted and replicated without a need for in-depth statistical modelling and that can be applied in various country contexts where Plasmodium falciparum transmission is reported. Additionally, we sought to create an expedited approach for targeting ITNs where the timeline to construct, map, and apply outputs occurred within an accelerated time period. The intended use of our outputs was to prioritise which local government areas (LGAs) should receive precedence in already scheduled ITN campaigns as well as identify LGAs that should receive additional quantities of ITNs (through additional support from donors) given public health emergencies such as the COVID-19 pandemic and presence of other risk factors, including disruptive events, notably flooding and armed conflict. Here, we use the outputs from Nigeria to illustrate our approach. We present a technical yet practical methodology that malaria control programmes can adapt to their needs, along with recommendations as to which indicators could be considered while planning vector control interventions such as ITN campaigns in the context of limited resources and health emergencies.

\section{Methods}

\section{Approach}

We developed a prioritization methodology that can be used for systematically selecting areas that need to be targeted in an appropriate sequence with ITN distributions while taking into consideration malaria transmission risks, available resources, ongoing emergencies, and other relevant factors. Whereas the 
exercise was conducted for Nigeria, we present here a general approach that can be adopted by any country or region experiencing malaria transmission that has: (1) access to subnational country-specific malaria intervention data; (2) a reasonably reliable COVID-19 surveillance system; (3) conducted a Demographic and Health Survey (DHS) or Malaria Indicator Survey (MIS) within the past three years; (4) access to open-source spatial layers featuring data pertaining to covariates such as $P$. falciparum environmental suitability indices, educational attainment, and population density; (5) available conflict and/or disruptive event data (if relevant); (6) insecticide resistance data; and (7) other country-specific information or data relevant to malaria risk. Using a combination of data derived from these elements, we were able to calculate risk scores for each administrative unit within a given country and rank these units to inform prioritization.

The basis of our methodology is derived from a strategy developed by Hanafi-Bojd et al., where a geographic information system (GIS)-based weighted arithmetic and multiplicative approach was used to categorize and rank administrative units based on malaria hazard and risk in the context of targeting interventions in Iran, which experiences $P$. vivax and $P$. falciparum transmission ${ }^{5}$. Additional conceptual frameworks and methodologies that identified vulnerability and potential hazards from systematic review and expert consultation which led to the creation of spatially explicit malaria risk maps were considered 6-8. Similar indicator weighting methodologies and the creation of a malaria vulnerability/risk index were employed. In lieu of utilizing weighted indicators derived from the coefficients of regression analysis ${ }^{6}$, indicator weights were calculated utilizing expert opinion surveys administered among regional and global field experts.

The preceding approaches were adapted for application in settings where $P$. falciparum is the predominant malaria parasite, while considering practical needs for additional malaria resources due to the COVID-19 pandemic. Therefore, $P$. falciparum-specific factors were utilised in calculating final risk scores, along with COVID-19 case burden and other factors that have impacts on malaria control. Unlike the approach used by Hanafi-Bojd et al., we focused on producing a single cumulative prioritization map (after quantifying and weighting multiple risk factor) for each of the country, as opposed to individual malaria risk and hazard maps. We did, however, combine similar drivers of falciparum malaria transmission with other malaria risk factors, including intervention coverage, population density, and presence and density of disruptive events. These risk factors were used to characterize administrative units according to their risk of malaria transmission based on their vulnerability for breeding and maintenance of malaria vectors, levels of intervention coverage, and social and biological susceptibility factors.

The steps below summarize the approach used to calculate the final risk score for each administrative unit (e.g., district, local government area, state, etc.). Step-by-step details of the implementation of the approach using the $\mathrm{R}$ software are provided under a separate section (Calculation of prioritization scores). 
- Most relevant risk factors that need to be considered for prioritization of planned vector control interventions (e.g., ITN campaign) were identified. The types and number of factors used for prioritization could differ between countries and interventions.

- The possible range of values of each factor were classified into a number of classes (typically four). For example, a factor such as "IRS implementation in 2020" has two classes ("Yes" and "No"), whereas "\% households with at least 1 ITN for 2 people" could have four classes (e.g., <35\%, 35-49\%, $50-67 \%$, and $>67 \%$ ). Where appropriate, the cut-off values were determined using "natural breaks" (where classes are based on natural groupings inherent in the data) as obtained using the R software (R Studio version 4.0.3).

- Rank values, defined here as whole numbers typically ranging from 1 to 4 (but can be 0 and 1 for a binary factor, denoting absence of presence of a particular intervention, for example), were assigned to each class of each factor. The higher the rank value the greater the malaria risk associated with that class. For example, the rank value of the class " $<35 \%$ " for the factor "\% households with at least 1 ITN for 2 people" mentioned above would be 4 whereas " $>67 \%$ " would be assigned a rank value of 1.

- A short survey questionnaire was circulated among malaria professionals and academics with malaria research experience to ask them to rank each factor on a scale of 0 - 10 in terms importance for quantifying malaria risk. An average rank was calculated for each factor, and then standardized on a scale of $0-1$ by dividing it by 10 .

- Each administrative unit was then assigned rank values for each factor, which was multiplied by the respective weight of the factor to obtain the risk scores. Risk scores of each factor were then summated to generate a composite prioritization score for each administrative unit.

Classification intervals featured in Table 1 were calculated using data from Nigeria as an example. We aim to provide a generalized approach that can be adopted in other counties that may have access to different types of data or may adopt different malaria control activities. For example, SMC is typically conducted only in limited regions, therefore this data would not be relevant for countries in other regions of Africa where it is not implemented.

\section{Data inputs}

The data related to each of the factors were obtained from various sources, including government documents, such as national malaria control strategic plans, ITN operational plans, vector control coverage data, and entomological and epidemiological reports. Literature covering models and approaches used to quantify and target ITNs in malaria endemic settings were also reviewed in detail ${ }^{5-8}$. These documents were used to inform the approach utilised for modelling and targeting ITN distributions.

A combination of environmental covariate data, Demographic and Health Survey (DHS) data, intervention coverage, internally displaced population data and predicted surface layers were used as the primary 
data inputs to generate prioritisation maps for Nigeria. A temperature suitability index for $P$. falciparum transmission was obtained from the Malaria Atlas Project (MAP). Mean annual rainfall and rainfall anomalies for each administrative unit were calculated using Climate Hazards Group InfraRed Precipitation with Station (CHIRPS) data. ITN coverage data was provided by Nigeria's National Malaria Elimination Programme (NMEP), along with projected population estimates for years 2019 and 2020. Built-up area presence data from the Global Human Settlement Layer (GHSL) Project produces global spatial information about the human presence on the planet over time and relies on automatic analysis of satellite imagery to produce fine-scale maps quantifying built-up structures in terms of their location and density and can be utilised. Built-up area presence was used as a proxy for classifying rural and urban areas. Intervention coverage (including ITN availability, or the proportion of households with at least 1 net per 2 people) was interpolated using survey data collected through the most recent DHS or MIS. Data from 2019 featuring distribution of internally displaced persons (IDPs) and high-risk populations (HRPs) per administrative unit was obtained from the Humanitarian Data Exchange.

\section{Spatial interpolation}

A major component that influenced our prioritization scheme was previous ITN depth of coverage, as obtained and spatially interpolated using data from DHS or MIS surveys. Using the R-INLA package a Bayesian inference spatial smoothing approach called Integrated Nested Laplace Approximation with Stochastic Partial Differential Equation (INLA-SPDE) was used to fit a geostatistical model predicting the proportion of households with at least 1 ITN per 2 de facto household population at unsampled locations. This process was conducted using cluster-level input data from the most recent DHS or MIS which collected information on household-level ITN ownership. DHS and MIS clusters were georeferenced and cluster-level estimates served as marked data points. DHS and MIS data generally allow for the calculation of representative estimates at the national, regional, and urban/rural levels, but the INLA process allows for pixel-level estimates which can be aggregated to establish subregional estimates based on available administrative boundaries. A logistic regression model was fit in INLA to estimate the probability of ITN availability across the mesh while accounting for spatial autocorrelation. Also included in the INLA function was an estimation stack comprised of the cluster-level ITN estimates as well as corresponding covariate estimates. Raster values were extracted for each DHS or MIS cluster point using a $2 \mathrm{~km}$ buffer for urban clusters and a $10 \mathrm{~km}$ buffer for rural clusters. The final output was a surface layer featuring predicted proportion of households with at least 1 ITN per 2 persons. This layer was combined with other factors, as listed in Table 1, to generate a final prioritization score for each administrative unit. The following section details how this was achieved.

\section{Calculation of prioritization scores}

Here we present a step-by-step framework illustrating our method for calculating prioritization scores in $\mathrm{R}$ Studio version 4.0.3, including only some of the important variables that could be used in most malaria 
endemic countries (especially in Africa) in Table 2. Other (country-specific) factors could be included in the calculation alongside the input variables described below. Syntax for each step, including a list of required packages for calculation of prioritization scores in $\mathrm{R}$ Studio, can be found in the GitHub repository listed in the supplementary files.

\section{Results}

The prioritization score calculation framework was used to create classifications for each of the 9 indicators (factors) featured in Table 1 and identify administrative units that fell within each factor's classification interval. Figure 1 a-j illustrates the geospatial distribution of each of the factors and corresponding classification, as well as the final prioritization map created using these inputs. We found that there was minimal geographic synchronicity between factors, excluding number of years since last distribution campaign (Fig. 1.d) and presence of internally displaced populations (Fig 1.i), and mean annual rainfall (Fig. 1.a) and P. falciparum temperature suitability index (Fig.1.b). The geospatial heterogeneity found among other risk factors supports the recommendation that a range of variables and covariates should be considered when using data to target ITN distributions, especially if implemented in a public health emergency or disease outbreak setting.

\section{Application of results}

Prioritization maps were shared with Nigeria's NMEP to illustrate which administrative units should be prioritised during upcoming ITN distribution campaigns as well as those that may need external financial support to achieve universal coverage (amidst the COVID-19 epidemic). Nigeria's ITN distribution plan focused on dissemination of ITNs over the course of four years (2020-2023). Figure 2. features spatial distribution of local government areas by prioritization class based on final prioritization scores (Figure 2.a.) compared to LGAs that fall within states targeted for distribution during the country's 2020 campaign (Figure 2.b.). It was recommended that LGAs with final prioritization scores that corresponded with risk classification of moderate-high, high, or extremely high risk be prioritised in initial years of distribution (2020 and 2021) and that that LGAs with final prioritization scores that corresponded with moderate, moderate-low, or low risk classifications be targeted in subsequent years (2022 and 2023).

For operational purposes state-level prioritization scores were also calculated. This was done by taking the mean weighted malaria risk score of all LGAs per state. This map (Figure 3.) featured was shared with the programme in the event that ITN distribution could not be targeted at the LGA level. The majority of LGAs and states with the highest prioritization scores were featured in the south of the country, where only a few regions were originally targeted for ITN distribution in 2020.

\section{Discussion}

Malaria risk factors that were given the largest influence on the final prioritization scheme included mean annual rainfall, number of years since last ITN distribution, and proportion of households with at least 1 
ITN per 2 people (standard weights ranging from $0.75-0.78$ ). In Nigeria, higher $P$. falciparum suitability index classification and increased mean annual rainfall correlated spatially with each other, as well as administrative units characterized as higher priority, indicating that our approach could potentially exclude one of these environmental factors as a data input. The most recent DHS survey indicated generally higher malaria prevalence rates in the northern compared to southern states, although climatic conditions in the latter are relatively more suitable for transmission. This could be due to differences in other factors that have not been accounted for, including socio-economic status and housing conditions. Nevertheless, most of the states where ITN campaigns have not been conducted for more than five years were located in the southern part of the country. Furthermore, SMC has been implemented only in the northern states in the Sahel region. Despite COVID-19 case counts contributing the least in standard weight (0.28) among all indicators, this factor corresponded with higher priority administrative units in southern Nigeria. The aforementioned factors aligned with expectations that southern regions of Nigeria would require increased prioritization for vector control interventions.

The spatially interpolated layer that features estimated ITN depth of coverage was a major component in informing regions that should be prioritizing in upcoming distributions. This layer does, however, require a certain amount of modelling to create, and therefore differs from the other factors that were used to inform targeting. Additionally, due to the depreciation of ITN effectiveness over time, DHS or MIS survey data that is older than 3 years should not be used to create the ITN depth of coverage layer. If survey data older than 3 years is used, a component that accounts for net decay should be integrated. In the event that this layer cannot be created, or the timeline for producing outputs is extremely limited, we are confident that the remaining factors alone could be used to inform targeting and prioritization during a public health emergency.

There was minimal spatial correlation between presence of internally displaced populations and administrative units characterized as higher priority; however, the presence of internally displaced populations was the lowest weighted (0.40) indicator, with the exception of the COVID-19 case counts. Although southern states have a more suitable climate for malaria transmission, malaria prevalence is actually higher in the north of Nigeria. This may be in part a result of the lower socio-economic status observed in this region. Despite higher transmission reported in the north, our outputs show that local government areas that were classified as high priority areas were located primarily in the south. The differentiation in prioritization region findings emphasize the potential importance of considering additional factors that do not directly influence malaria transmission, such as active epidemic-related case counts, and previous and current intervention coverage. Future renditions of the model could also consider the role of socioeconomic status, if relevant.

This strategy could be adapted and applied in a variety of public health emergency and disease outbreak settings. The rationale for including COVID-19 case counts as a prioritization factor was due to the influx of inpatients admitted to health centres and hospitals and subsequent need for additional ITNs in facilities through emergency distribution. This factor could therefore be changed to represent distribution of other diseases of outbreak potential. 
Whereas this approach was initially designed for targeting of ITN distributions amidst a public health emergency, its scope could be adapted for targeting of a wider range of vector control interventions to optimize the use of limited resources, especially in regions experiencing increased insecticide resistance and the need to use expensive tools and strategies.

\section{Limitations}

Data on COVID-19 case counts were not available for administrative level 2 (LGA), however, COVID-19 case counts were available for administrative level 1 (state). We therefore assigned the state-level COVID19 specific risk category to all LGAs that fell within a given state. LGA-level data on distribution of community health workers (CHWs) offering malaria test and treat services was not available and was therefore not included as an input in calculation risk and final prioritization scores. Indicators such as test positivity rate, annual blood examination rate, and malaria prevalence were not included in the analysis for generating prioritization maps as malaria morbidity data collected through routine facility-based malaria surveillance was believed to not accurately represent case burden. We therefore relied primarily on environmental covariates and malaria intervention coverage to assess malaria risk. Given the need to quickly produce outputs to inform distribution during the COVD-19 pandemic, in-depth evaluation of the accuracy of prediction of malaria risk on environmental factors, as well as the impact of inclusion or exclusion of some factors on the prioritization index was not conducted.

\section{Conclusions}

This paper presents a decision-making tool that can be used to prioritise regions for mass or emergency ITN distribution, especially during public health emergency situations. Whereas there are some limitations in terms of the factors included to prioritise regions for ITN distribution in Nigeria, we believe that we have created a valuable tool for ITN targeting during a public health emergency as it can be used to produce outputs in a limited time frame, while using a range of indicators that are typically available and systematically collected in countries that experience $P$. falciparum transmission. Results from this exercise suggest that factors outside of those that exclusively characterize risk of malaria transmission and maintenance of malaria vectors, for example, concurrent intervention coverage and presence of disruptive events, should also be considered when targeting ITN distributions. Not only do we present a framework that can be adapted to variety of country-contexts, but we also provide a baseline methodology that could be adjusted for targeting a wider range vector control interventions.

\section{Abbreviations}

CHIRPS Climate Hazards Group InfraRed Precipitation with Station

CHW Community Health Worker

DHS Demographic Health Survey 
GHSL Global Human Settlement Layer

GHSL-SMOD Global Human Settlement Layer Model Grid

GIS Geographic Information System

INLA-SPDE Integrated Nested Laplace Approximation with Stochastic Partial Differential Equation

IRS Indoor Residual Spraying

ITN Insecticide Treated Net

LGA Local Government Area

LST Land Surface Temperature

MAP Malaria Atlas Project

MIS Malaria Indicator Survey

$\mathrm{MOH}$ Ministry of Health

NMCP National Malaria Control Programme

NMEP National Malaria Elimination Programme

PBO Piperonyl Butoxide

SMC Seasonal Malaria Chemoprophylaxis

\section{Declarations}

\section{Ethics approval and consent to participate}

Not applicable

\section{Consent for publication}

Not applicable

\section{Availability of data and materials}

The datasets used and/or analysed during the current study are available from the corresponding author on reasonable request. 


\section{Competing interests}

The authors declare that they have no competing interests.

\section{Funding}

This work was supported by GiveWell.

\section{Authors' contributions}

A.Y., W.E, H.H. and T.A. conceived the presented idea. A.Y., W.E, and H.H. developed the approach and methodology. A.Y. and W.E. performed all computation, data extraction, analysis and mapping. M.W. developed the approach for spatial interpolation and provided code and technical assistance in creating all spatially interpolated layers. A.Y. and W.E wrote the manuscript with support from M.W. and T.A. T.A. and J.T. supervised the project. M.K. and B.M.A. provided Nigeria-related data and provided technical advice. M.M-R. and C.N. provided technical advice and facilitated the study.

\section{Acknowledgements}

We would like to thank Olusola Oresanya (Malaria Consortium Nigeria), Philip Oyale (National Malaria Elimination Programme, Nigeria), Anthony Nuwa (Malaria Consortium Uganda), Jimmy Opigo (National Malaria Control Programme, Uganda), and Maria Rodrigues (Malaria Consortium Mozambique) for their technical advice. The study was funded by GiveWell.

\section{Authors' information (optional)}

\section{References}

1. Hemingway J, Shretta R, Wells TNC, et al. Tools and Strategies for Malaria Control and Elimination: What Do We Need to Achieve a Grand Convergence in Malaria? PLoS Bio. I 2016, doi:10.1371/journal.pbio.1002380

2. Stresman G, Bousema T, Cook J. Malaria Hotspots: Is There Epidemiological Evidence for Fine-Scale Spatial Targeting of Interventions? Trends Parasitol. 2019, 35(10):822-834. doi:10.1016/j.pt.2019.07.013

3. Carter R, Mendis KN, Roberts D. Spatial targeting of interventions against malaria. Bull World Health Organ. 2000, 78(12):1401-1411. doi:10.1590/S0042-96862000001200007

4. Diptyanusa A, Zablon KN. Addressing budget reduction and reallocation on health-related resources during COVID-19 pandemic in malaria-endemic countries. Malar J. 2020, 19(1):411. 
doi:10.1186/s12936-020-03488-y

5. Hanafi-Bojd AA, Vatandoost $\mathrm{H}$, Oshaghi MA, et al. Spatial analysis and mapping of malaria risk in an endemic area, south of Iran: A GIS based decision making for planning of control. Acta Trop. 2012, 122(1):132-137. doi:10.1016/j.actatropica.2012.01.003

6. Hagenlocher M, Castro MC. Mapping malaria risk and vulnerability in the United Republic of Tanzania: A spatial explicit model. Popul Health Metr. 2015, 13(1):2. doi:10.1186/s12963-015-00362

7. Lei L, Richards JS, Li ZH, Gong YF, Zhang SZ, Xiao N. A framework for assessing local transmission risk of imported malaria cases. Infect Dis Poverty. 2019, 8(1):43. doi:10.1186/s40249-019-0552-6

8. Hashiguchi L. Uganda: Evidence for a Targeted Malaria Response., 2018.

\section{Tables}

Table 1 - List of ITN distribution prioritization factors and corresponding method of extraction, weight, classification, rank, and risk characterization for Nigeria 


\begin{tabular}{|c|c|c|c|c|c|}
\hline Factor & $\begin{array}{l}\text { Method of } \\
\text { extraction/application }\end{array}$ & $\begin{array}{l}\text { Standard } \\
\text { Weight }\end{array}$ & Classification & $\begin{array}{l}\text { Rank } \\
\text { value }\end{array}$ & $\begin{array}{l}\text { Risk } \\
\text { characterization }\end{array}$ \\
\hline \multirow{4}{*}{$\begin{array}{l}\text { CHIRPS } \\
\text { mean } \\
\text { annual } \\
\text { rainfall } \\
\text { (mm) from } \\
\text { May } 2019 \text { to } \\
\text { April } 2020\end{array}$} & \multirow{4}{*}{$\begin{array}{l}\text { Climate Hazards Group } \\
\text { InfraRed Precipitation with } \\
\text { Station (CHIRPS) data was } \\
\text { used to calculate mean } \\
\text { monthly rainfall (from May } \\
2019 \text { to April 2020) by } \\
\text { administrative boundary }\end{array}$} & \multirow[t]{4}{*}{0.78} & > 207 mm & 4 & Very high \\
\hline & & & $\begin{array}{l}148-207 \\
\mathrm{~mm}\end{array}$ & 3 & High \\
\hline & & & $92-147 \mathrm{~mm}$ & 2 & Moderate \\
\hline & & & $\leq 91 \mathrm{~mm}$ & 1 & Low \\
\hline \multirow{4}{*}{$\begin{array}{l}\text { P.f. } \\
\text { temperature } \\
\text { suitability } \\
\text { index }\end{array}$} & \multirow{4}{*}{$\begin{array}{l}\text { Extracted from raster layer } \\
\text { created by Malaria Atlas } \\
\text { Project (MAP). }\end{array}$} & \multirow[t]{4}{*}{0.58} & $\leq 0.453$ & 1 & Low \\
\hline & & & $0.454-0.570$ & 2 & Moderate \\
\hline & & & $0.571-0.680$ & 3 & High \\
\hline & & & $>0.680$ & 4 & Very high \\
\hline \multirow{4}{*}{$\begin{array}{l}\text { COVID-19 } \\
\text { cases } \\
\text { reported as } \\
\text { of Date X. }\end{array}$} & \multirow{4}{*}{$\begin{array}{l}\text { Aggregated to respective } \\
\text { administrative unit using } \\
\text { data from ministry of } \\
\text { health }(\mathrm{MOH}) \text { COVID-19 } \\
\text { dashboards or external } \\
\text { international reporting } \\
\text { sources. Values provided } \\
\text { here are representative of } \\
\text { case counts in Nigeria as } \\
\text { of July } 21,2020 \text {. }\end{array}$} & \multirow[t]{4}{*}{0.28} & 5,094 & 4 & Very high \\
\hline & & & $\begin{array}{l}3,091- \\
5,094\end{array}$ & 3 & High \\
\hline & & & $\begin{array}{l}1,143- \\
3,090\end{array}$ & 2 & Moderate \\
\hline & & & $<1,143$ & 1 & Low \\
\hline \multirow{4}{*}{$\begin{array}{l}\text { Number of } \\
\text { years since } \\
\text { last mass } \\
\text { ITN } \\
\text { distribution }\end{array}$} & \multirow{4}{*}{$\begin{array}{l}\text { Calculated for each } \\
\text { administrative unit using } \\
\text { dates reported in NMCP } \\
\text { documents. }\end{array}$} & \multirow[t]{4}{*}{0.75} & $\geq 6$ years & 4 & Very high \\
\hline & & & 5 years & 3 & High \\
\hline & & & 3 years & 2 & Moderate \\
\hline & & & $<3$ years & 1 & Low \\
\hline \multirow{2}{*}{$\begin{array}{l}\text { PBO net } \\
\text { distribution } \\
\text { in } 2019\end{array}$} & \multirow{2}{*}{$\begin{array}{l}\text { Obtained from NMCP } \\
\text { operational plans and } \\
\text { intervention coverage } \\
\text { documents. }\end{array}$} & \multirow[t]{2}{*}{0.53} & $\begin{array}{l}\text { Not } \\
\text { distributed }\end{array}$ & 1 & High \\
\hline & & & Distributed & 0 & Low \\
\hline \multirow{4}{*}{$\begin{array}{l}\text { Proportion } \\
\text { of } \\
\text { households } \\
\text { with at least } \\
1 \text { ITN per } 2 \\
\text { people }\end{array}$} & \multirow{4}{*}{$\begin{array}{l}\text { Modelled using INLA-SPDE } \\
\text { method on } 2018 \text { DHS } \\
\text { data. Values provided here } \\
\text { are Nigeria specific. }\end{array}$} & \multirow[t]{4}{*}{0.75} & $23 \%$ & 4 & Very high \\
\hline & & & $23-34 \%$ & 3 & High \\
\hline & & & $35-48 \%$ & 2 & Moderate \\
\hline & & & $48 \%$ & 1 & Low \\
\hline \multirow{2}{*}{$\begin{array}{l}\text { SMC } \\
\text { coverage in } \\
2019\end{array}$} & \multirow{2}{*}{$\begin{array}{l}\text { Obtained from NMCP } \\
\text { operational plans and } \\
\text { intervention coverage } \\
\text { documents. }\end{array}$} & \multirow[t]{2}{*}{0.82} & $\begin{array}{l}\text { Not } \\
\text { implemented }\end{array}$ & 1 & High \\
\hline & & & Implemented & 0 & Low \\
\hline Built up area & Extracted and aggregated & 0.54 & 0.0085 & 4 & Very high \\
\hline
\end{tabular}




\begin{tabular}{|c|c|c|c|c|c|}
\hline \multirow{3}{*}{$\begin{array}{l}\text { presence } \\
\text { index (proxy } \\
\text { for } \\
\text { urban/rural } \\
\text { designation) }\end{array}$} & \multirow[t]{3}{*}{$\begin{array}{l}\text { to respective } \\
\text { administrative unit using } \\
\text { SMOD raster layers. }\end{array}$} & & $\begin{array}{l}0.051 \\
-0.0084\end{array}$ & 3 & High \\
\hline & & & $0.76-0.05$ & 2 & Moderate \\
\hline & & & 0.76 & 1 & Low \\
\hline \multirow{2}{*}{$\begin{array}{l}\text { Internally } \\
\text { displaced } \\
\text { populations } \\
\text { (resulting } \\
\text { from armed } \\
\text { conflict) in } \\
2020\end{array}$} & \multirow[t]{2}{*}{$\begin{array}{l}\text { Obtained from data sets } \\
\text { provided by Humanitarian } \\
\text { data exchange. }\end{array}$} & \multirow[t]{2}{*}{0.40} & Present & 1 & High \\
\hline & & & Not present & 0 & Low \\
\hline
\end{tabular}

Table 2 - List of steps required to create final prioritization maps, and corresponding sources of relevant external data 


\begin{tabular}{|lll|}
\hline Step & Description & External File Source (if relevant) \\
\hline 2 & $\begin{array}{l}\text { Load relevant R packages and } \\
\text { libraries }\end{array}$ & N/A \\
& $\begin{array}{l}\text { Obtain administrative boundaries of } \\
\text { country/region of interest by either } \\
\text { loading a pre-existing shapefile or } \\
\text { directly through GADM }\end{array}$ & gadm.org/maps \\
\hline $3 \quad \begin{array}{l}\text { Obtain water boundary shapefile } \\
\text { layers from OCHA Humanitarian } \\
\text { Data Exchange (if relevant). }\end{array}$ & https://data.humdata.org/ \\
\hline 4 & $\begin{array}{l}\text { Obtain temperature suitability index } \\
\text { raster layer from Malaria Atlas } \\
\text { Project. If a temperature suitability } \\
\text { index raster is not available or } \\
\text { accessible, a combination of mean } \\
\text { monthly rainfall, land surface } \\
\text { temperature, and elevation can be } \\
\text { used. }\end{array}$ \\
\hline
\end{tabular}

5 Obtain monthly mean rainfall raster https://data.chc.ucsb.edu/products/CHIRPS-2.0/ layers from CHIRPS

6 Import spatially interpolated raster layer featuring proportion of households with at least 1 ITN per 2 people (if available). This is created using cluster-level DHS or MIS data pertaining to ITN use and raster layers of covariates such as travel time to nearest city of 60,000 or more inhabitants, 2015 educational attainment for women of reproductive age, 2015 prevalence of improved housing, and the 2015 settlement model (a combination of built environment and population density), via the INLA-SPDE package.

7 Aggregate relevant indicators (see Table 1 for examples) to desired administrative boundary level and add to a master dataset that is linked to the name and unique code for the desired administrative boundary. The unit which data is aggregated to is dependent upon the resolution of intervention data available (for example number of persons receiving SMC per local government area). Ensure that desired administrative boundary shapefile exists prior $t$ aggregating values to administrative level.

DHS cluster data available upon request from:

https://dhsprogram.com/data/availabledatasets.cfm

Covariate layers database:

https://malariaatlas.org/explorer/\#/

N/A

Page 16/20 


\begin{tabular}{|l|l|}
\hline 8 & $\begin{array}{l}\text { Convert categorical variable to most } \\
\text { appropriate categorical variables } \\
\text { from literature review and/or use } \\
\text { natural breaks from getJenksBreaks } \\
\text { function in R with desired number of } \\
\text { classes }\end{array}$ \\
\hline $\begin{array}{l}\text { Assign rank values to indicators, } \\
\text { keeping in mind that the } \\
\text { complement (or inverse) of some } \\
\text { indicators may need to be calculated } \\
\text { in order to maintain consistency with } \\
\text { increased or decreased risk scores. } \\
\text { For example, increased rainfall } \\
\text { values (larger positive value) may } \\
\text { imply increased malaria risk while, } \\
\text { high population density, high } \\
\text { urbanization (larger positive value) } \\
\text { may imply decreased malaria risk } \\
\text { especially if the main vector's } \\
\text { preferred larval habitats are in rural } \\
\text { settings }\end{array}$ \\
$\begin{array}{l}\text { Multiply standard weight value (as } \\
\text { featured in Table 1) by rank values } \\
\text { for each factor to create factor- } \\
\text { specific score. }\end{array}$ \\
\hline $\begin{array}{l}\text { Summate final factor-specific risk } \\
\text { scores for each administrative unit } \\
\text { so that each administrative } \\
\text { boundary has cumulative } \\
\text { prioritization score where a higher } \\
\text { total score is indicative of higher } \\
\text { prioritization for ITN targeting }\end{array}$ \\
\hline 11 N/A \\
\hline
\end{tabular}

\section{Figures}




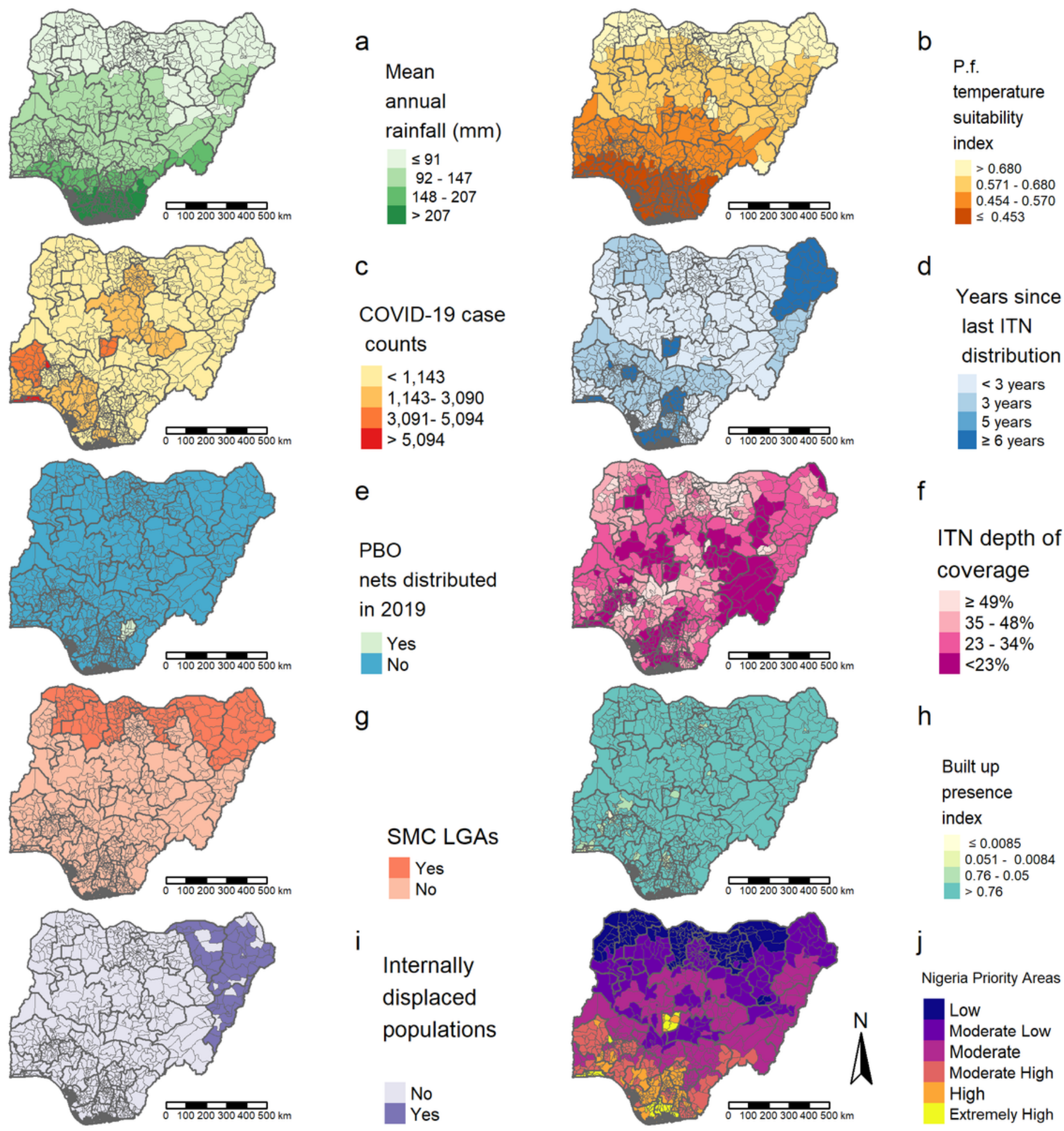

\section{Figure 1}

Geospatial risk classification of all input factors (a-i) used to create final prioritization map (j) Note: The designations employed and the presentation of the material on this map do not imply the expression of any opinion whatsoever on the part of Research Square concerning the legal status of any country, territory, city or area or of its authorities, or concerning the delimitation of its frontiers or boundaries. This map has been provided by the authors. 

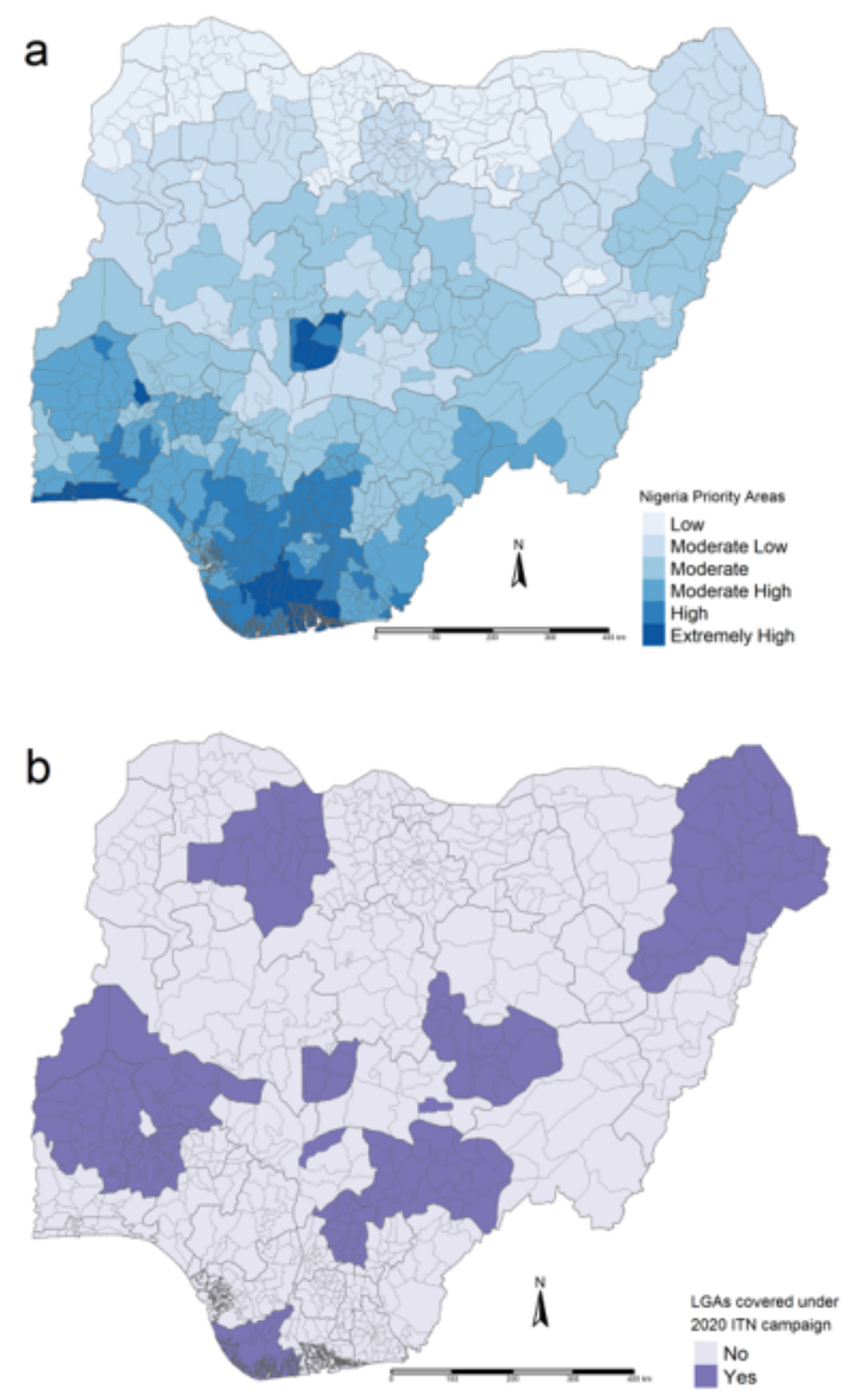

Figure 2

Spatial distribution of LGAs by prioritization class (a) compared to distribution of LGAs covered under current 2020 ITN distribution plan (b) Note: The designations employed and the presentation of the material on this map do not imply the expression of any opinion whatsoever on the part of Research Square concerning the legal status of any country, territory, city or area or of its authorities, or concerning the delimitation of its frontiers or boundaries. This map has been provided by the authors. 


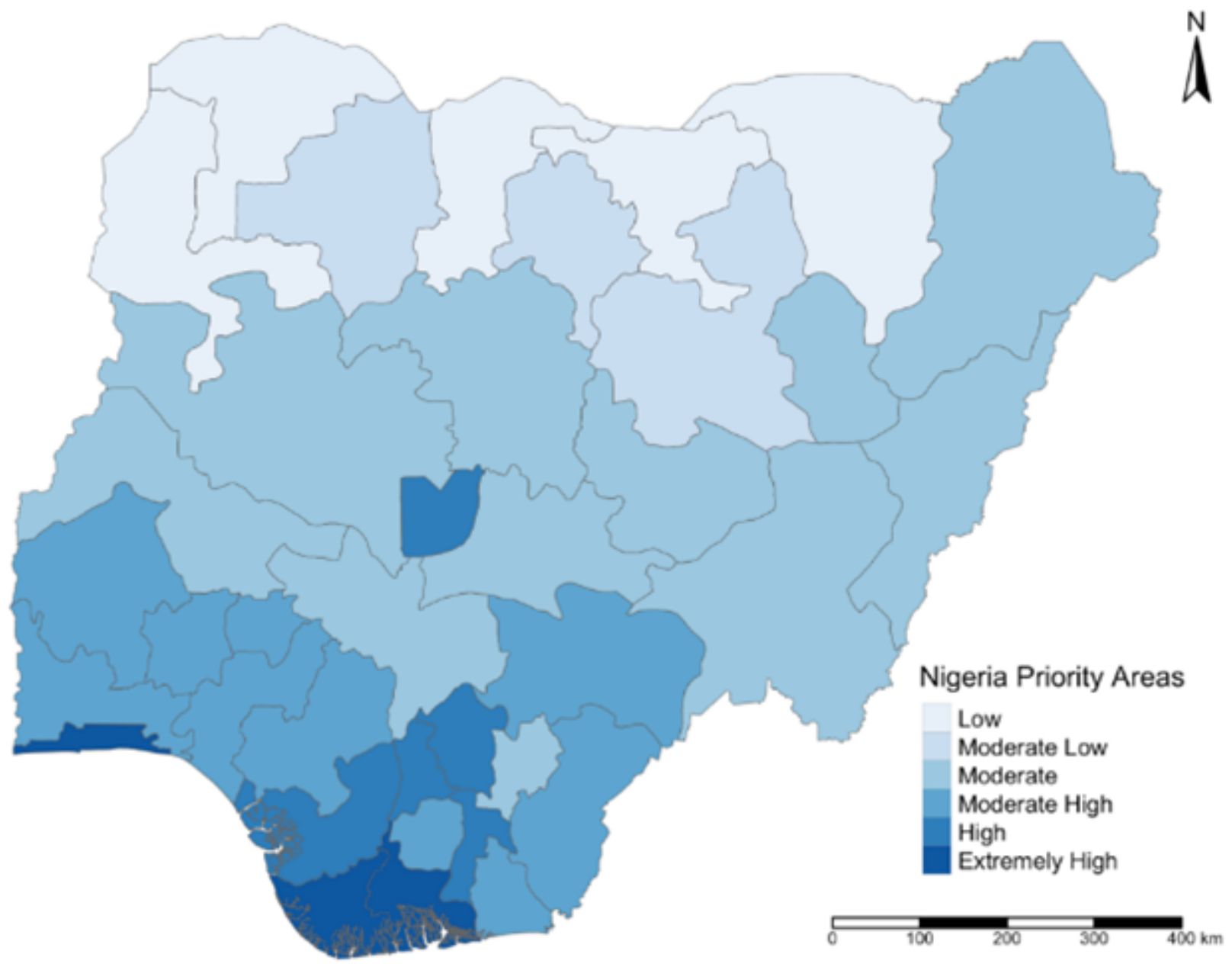

Figure 3

State-level prioritization scheme Note: The designations employed and the presentation of the material on this map do not imply the expression of any opinion whatsoever on the part of Research Square concerning the legal status of any country, territory, city or area or of its authorities, or concerning the delimitation of its frontiers or boundaries. This map has been provided by the authors.

\section{Supplementary Files}

This is a list of supplementary files associated with this preprint. Click to download.

- Additionalfiles.docx 